\title{
Seasonal Predictability of the North Atlantic Oscillation and Zonal Mean Fields Associated with Stratospheric Influence in JMA/MRI-CPS2
}

\author{
Naoaki Saito ${ }^{1}$, Shuhei Maeda ${ }^{2}$, Tosiyuki Nakaegawa ${ }^{1}$, Yuhei Takaya ${ }^{1}$, \\ Yukiko Imada ${ }^{1}$, and Chihiro Matsukawa ${ }^{3}$ \\ ${ }^{1}$ Climate Research Department, Meteorological Research Institute, Tsukuba, Japan \\ ${ }^{2}$ Climate Prediction Division, Japan Meteorological Agency, Tokyo, Japan \\ ${ }^{3}$ Numerical Prediction Division, Japan Meteorological Agancy, Tokyo, Japan
}

\begin{abstract}
The North Atlantic Oscillation (NAO) is the most dominant hemispheric variability affecting the winter climate. It is, however, difficult to predict the NAO on a seasonal time scale. Thus, a better understanding of the NAO is important for improving the accuracy of seasonal forecasts. We investigated the seasonal predictability of the NAO and the zonal mean fields of the stratosphere using hindcasts based on the operational seasonal prediction system of the Japan Meteorological Agency. We found that both the predictive skill and the potential predictability of the NAO index increased from late winter to early spring and that this seasonality relates to high predictability of the zonal mean geopotential height in the stratosphere and near the surface. Analysis of the convergence/divergence of the Eliassen-Palm flux indicates that the high predictability in the stratosphere in late winter results from the large signal of upward-propagating Rossby waves. The downward influence of the predictable signal from the stratosphere to the troposphere was also quantitatively estimated using the approach proposed by Ambaum and Hoskins (2002). The signal of the surface pressure estimated from that of stratospheric potential vorticity is roughly consistent with the actual signal, substantiating the stratospheric contribution to the near-surface seasonal predictability.
\end{abstract}

(Citation: Saito, N., S. Maeda, T. Nakaegawa, Y. Takaya, Y. Imada, and C. Matsukawa, 2017: Seasonal predictability of the North Atlantic Oscillation and zonal mean fields associated with stratospheric influence in JMA/MRI-CPS2. SOLA, 13, 209-213, doi:10.2151/sola.2017-038.)

\section{Introduction}

The accuracy of current seasonal forecasts relies on the signals in atmospheric variability associated with tropical ocean variability on a seasonal to interannual time scale (Palmer and Hagedorn 2006). On the other hand, the predictability of the North Atlantic Oscillation (NAO; Barnston and Livezey 1987) or the Arctic Oscillation (AO; Thompson and Wallace 1998), which are the most dominant forms of hemispheric variability in boreal winters (hereafter seasons refer to those for the Northern Hemisphere), was until recently thought to be low on a seasonal time scale (Johansson 2007), limiting the winter forecasting capability.

However, some studies have pointed out that the stratosphere is one of the sources of predictability of the tropospheric variability (Kuroda 2008; Marshall and Scaife 2010; Sigmond et al. 2013) and contributes to the enhancement of the predictive skill of intraseasonal to interannual variability, including the NAO/ AO (Scaife et al. 2014; Scaife et al. 2016). Sigmond et al. (2013) showed that seasonal forecast skill in the troposphere is enhanced when the forecast is initialized during stratospheric sudden warm-

Corresponding author: Naoaki Saito, Meteorological Research Institute, 1-1 Nagamine, Tsukuba, Ibaraki 305-0052, Japan. E-mail: saito@mri-jma. go.jp. C2017, the Meteorological Society of Japan. ings (SSWs). Kuroda (2008) compared numerical forecasts using models with and without a model stratosphere and showed that tropospheric forecast skill is enhanced on extended-range time scale when the forecast model top extends above $40 \mathrm{hPa}$. Marshall and Scaife (2010) showed that enhanced model stratospheric resolution leads to earlier capture of SSW events than standard model and improves surface forecasts. Scaife et al. (2014) presented a high predictive skill for the NAO in winter (December to February) using the Met Office Global Seasonal forecast System 5 (GloSea5) with both a high model top at $85 \mathrm{~km}$ and a high ocean resolution and Scaife et al. (2016) showed that this high skill for the NAO was conditional on the inclusion of SSWs in the ensemble. These results suggest that the stratosphere affects the predictive skill of the NAO variability at the surface.

The mechanisms of the downward influence from the stratosphere to the troposphere have been extensively studied and drawn a great deal of attention owing to the implications of improving seasonal predictions. For example, Baldwin and Dunkerton (2001) revealed that the signals of the polar vortex propagate downward from the stratosphere to the troposphere over one to two months. The mechanisms of the downward influence are still contentious, though some theories have been proposed: wave-mean flow interaction (Kuroda and Kodera 1999), reflections of planetary waves due to the change in stratospheric circulation (Perlwitz and Harnik 2003; Perlwitz and Harnik 2004; Kodera et al. 2008), influence of the stratospheric potential vorticity (PV) anomaly (Ambaum and Hoskins 2002), and so on (please refer to Kidston et al. 2015 for a holistic review). Among these, Ambaum and Hoskins (2002; hereafter AH02) suggested a mechanism in which the modulation of the stratospheric circulation affects the troposphere via geostrophic adjustment. They also provided quantitative estimates of the downward influence. Recently, Takemura and Maeda (2016) have quantitatively estimated the downward influence of the stratospheric composite anomalies on the troposphere during El Niño conditions based on AH02's approach.

Although several studies investigated and proposed dynamical mechanisms of downward influence of the stratosphere and presented the successful seasonal prediction of the NAO, fundamental dynamical processes connected to the high predictability have not been explicitly investigated using numerical models. In this study, we analyze the predictive skill and potential predictability of the NAO and their seasonality, focusing on the relation with the stratosphere. We use the Japan Meteorological Agency/Meteorological Research Institute-Coupled Prediction System version 2 (JMA/MRI-CPS2; Takaya et al. 2017), which is the latest operational seasonal prediction system of the JMA. In addition, we quantitatively estimated the downward influence of the signal from the stratosphere to the troposphere based on the approach by AH02 and Takemura and Maeda (2016).

\section{Data and methods}

\subsection{Data}

In terms of the NAO and the stratospheric variability, we analyzed a set of hindcasts of the JMA/MRI-CPS2 (Takaya et al. 
Table 1. Initial dates of the hindcast for the JMA/MRI-CPS2 from October to April (quoted from Takaya et al. 2017).

\begin{tabular}{cc}
\hline Initial month & Initial dates \\
\hline October & 13 September, 28 September \\
November & 13 October, 28 October \\
December & 12 November, 27 November \\
January & 12 December, 27 December \\
February & 16 January, 31 January \\
March & 10 February, 25 February \\
April & 12 March, 27 March \\
\hline
\end{tabular}

2017) and a long-term climate analysis, the Japanese 55-year Reanalysis (JRA-55; Kobayashi et al. 2015). The JMA/MRICGCM2 model top, the atmospheric-land-ocean-sea ice coupled model part of the JMA/MRI-CPS2, was set to $0.1 \mathrm{hPa}$ (about $64 \mathrm{~km}$ ), which is above the stratopause. The ensemble size of the hindcast was 10 for every initial month (initial dates are given in Table 1). We analyzed the hindcast data of the first three months during the 30-year period between 1981 and 2010. For example, we analyzed three-month forecasts for February to April that started from January 16 and 31. Although the weather forecast predictability might have some effects on the forecast skills analyzed here, we also obtained consistent results for the case of onemonth lead time (figure omitted). The NAO index was calculated as the difference between the normalized sea-level pressures measured in Lisbon, Portugal, and Stykkisholmur, Iceland, based on the definition by Hurrell (1995). We also evaluated the predictive skills for geopotential height $(\mathrm{Z})$ and the convergence/divergence of the Eliassen-Palm (EP) flux as physical quantities related to stratospheric circulation. The EP flux was calculated using Eqs. (3.1a), (3.1b), and (3.2) from Edmon et al. (1980).

\subsection{Methods}

The temporal anomaly correlation coefficient (ACC) between time-series of the ensemble mean forecast and those of the JRA-55 were used to evaluate the predictive skill. In the calculation, the anomalies of the forecasts and the JRA-55 with respect to their 30 -year averages were computed.

We assessed the potential predictability using the ensemble data according to Rowell et al. (1995) and Sugi et al. (1997). The authors devised a way to estimate potential predictability using amplitudes of both the signal and noise calculated from the ensemble mean and spread. We adopted this technique to assess the seasonal predictability of stratospheric and tropospheric variability. The variance of all ensemble members can be decomposed to the variance of the ensemble mean, which corresponds to deterministically predictable variability (signal), and the variance of the anomalies from the ensemble mean, which corresponds to unpredictable variability (noise). Here, we refer to the ratio of the variance of the signal to the total variance (signal plus noise) as an indicator of the potential predictability, which is defined as follows:

$$
R=\frac{S^{2}}{S^{2}+N^{2}}
$$

where $R$ is the potential predictability (signal-to-noise ratio) and $S^{2}$ and $N^{2}$ are the variances of the signal and noise, respectively. The calculations of $S^{2}$ and $N^{2}$ are given in the supplemental material. If the forecast model is perfect and ensemble size is large enough, the correlation coefficient between one ensemble member and the ensemble mean is represented by $\sqrt{R}$ (Sugi et al. 1997). Therefore, assuming that the model is perfectly able to predict the signal accurately and the ensemble method is perfectly able to estimate the noise, the correlation coefficient between the observation and the ensemble mean (predictive skill) is represented by $\sqrt{R}$. In reality, both the model and the ensemble method are imperfect, so predictive skill does not necessarily coincide with $\sqrt{R}$. Especially for the seasonal prediction of the NAO, the ACC is sometimes higher than $\sqrt{R}$ (Eade et al. 2014). However, the tendency of seasonality of $\sqrt{R}$ roughly coincides with that of the ACC (as shown later), which can be explained by the seasonality of $S^{2}$ and $N^{2}$, therefore $\sqrt{R}$ is worth assessing as an indicator of the potential predictability in our prediction system.

In the next section, we present seasonality and time-vertical structures of the ACC and $\sqrt{R}$ for the NAO index, geopotential height, and convergence/divergence of the EP flux, and we evaluate the forecast, comparing the ACC and $\sqrt{R}$.

\section{Results}

\subsection{Seasonality of predictability and forecast skill for the NAO index}

The seasonality of the ACC and $\sqrt{R}$ for the NAO index are shown in Fig. 1. The variability of $\sqrt{R}$ and the ACC showed a similar tendency: they both increased gradually from autumn and reached a maximum in late winter or spring. For the winter period (from December to February; DJF), the ACC was about 0.2, which is lower than that obtained by Scaife et al. (2014); meanwhile, ACCs were relatively high for February to April (FMA) and March to May (MAM).

\subsection{Predictability and forecast skill for geopotential height}

Here, we discuss the seasonality of the predictability of the $\mathrm{NAO}$ in terms of its relation with the stratosphere. Since the NAO is closely related to a hemispheric annular-like mode, AO, we examined the predictability of zonal mean fields.

The time-height cross-sections of $70^{\circ} \mathrm{N}-90^{\circ} \mathrm{N}$ averaged $\sqrt{R}$ and the ACC for the zonal mean field of geopotential height $\mathrm{Z}$ are shown in Figs. $2 a$ and $2 b$, respectively. The variations in the zonal mean $\mathrm{Z}$ averaged across this latitude band reflect the polar vortex strength. $\sqrt{R}$ and the ACC show similar structures, as is the case with the NAO index. From late winter (FMA) to spring (MAM), there are areas with fairly high predictability $(\sqrt{R})$ in the lower stratosphere between 50 and $100 \mathrm{hPa}$ and areas with moderately high predictability in the lower troposphere near the surface. This seasonality was consistent with the NAO index. In contrast, predictability in the upper troposphere (around $500 \mathrm{hPa}$ ) was lower than those for the stratosphere and the near surface. We consider that this local minimum of the predictability is due to the noise on a seasonal time scale caused by the breaking of the shortwavelength Rossby waves, which cannot intrude into the stratosphere. For AMJ, $\sqrt{R}$ and the ACC are still large in the stratosphere, while decreasing near the surface. The former is because the stratosphere is stable and persistent after the spring. The latter is because the connection between the stratosphere and the troposphere weakens after the spring. The stratosphere-troposphere coupling is active from November until late April when the polar

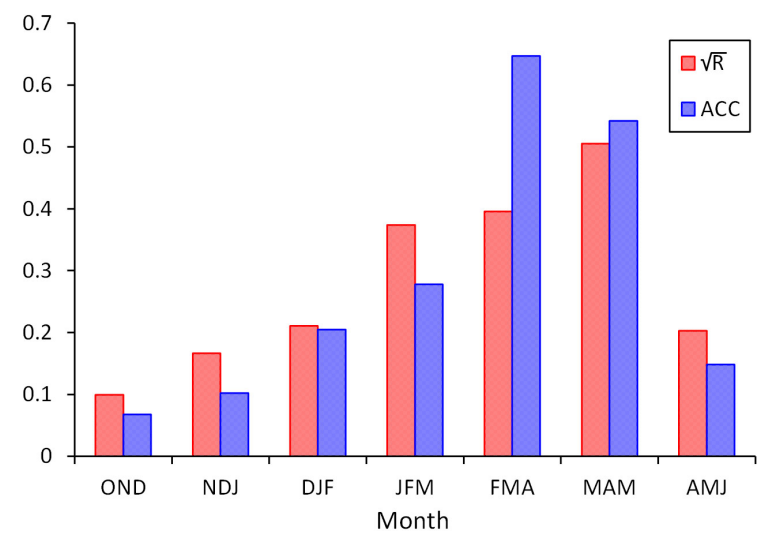

Fig. 1. Seasonality of the forecast skill ACC (blue bars) and its indicator $\sqrt{R}$ (red bars), the square root of the index of potential predictability for the NAO index. The horizontal axis indicates three-month averages (from OND to AMJ). 
vortex weakens in the Northern Hemisphere (e.g., Kidston et al. 2015; Hitchcock et al. 2014).

In order to find out the contributions of $N$ and $S$ to the seasonality of $\sqrt{R}$, seasonal changes of $N$ (the standard deviation of the noise) and $S$ (the standard deviation of the signal) at each altitude corresponding to $\sqrt{R}$ (Fig. 2a) are also shown in Figs. 2c and 2d, respectively. $N$ mostly increased in the stratosphere during autumn, reaching a maximum in DJF to JFM, and then decreased. On the other hand, after reaching a local minimum in NDJ, $S$ increased in the stratosphere from winter to early spring following $N$. In FMA, $S$ was nearly equal to $N$ in the lower stratosphere. The faster increase and slower decrease of $S$ than $N$ lead to the high predictability in early spring. These characteristics are the same as those observed in the troposphere. Especially near the surface, $S$ reached a maximum in FMA.

\subsection{Predictability of convergence/divergence of the Eliassen- Palm flux and its relation to the predictability of $Z$}

Since the zonal mean stratospheric circulation in the polar region is closely related to planetary waves propagating from the troposphere, the above-mentioned seasonality of the predictability in the stratosphere is also closely related to the waves. Figures $3 \mathrm{a}$, $3 \mathrm{~b}$, and $3 \mathrm{c}$ show time-height cross-sections of $45^{\circ} \mathrm{N}-75^{\circ} \mathrm{N}$ averaged $\sqrt{R}, N$, and $S$ for the convergence/divergence of the EP flux, respectively. The EP flux convergence/divergence represents the effect of the Rossby wave forcing on the zonal flow.
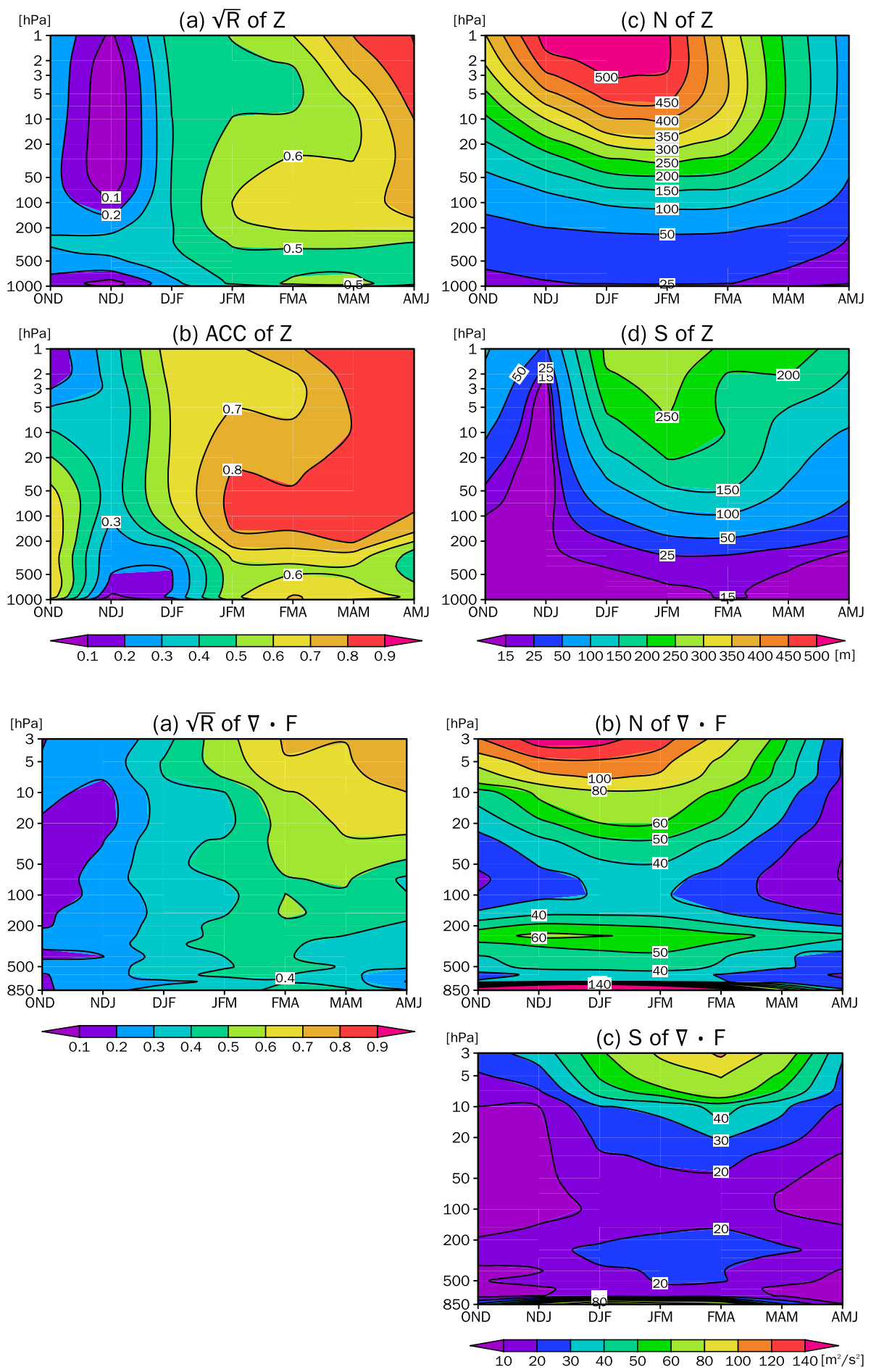
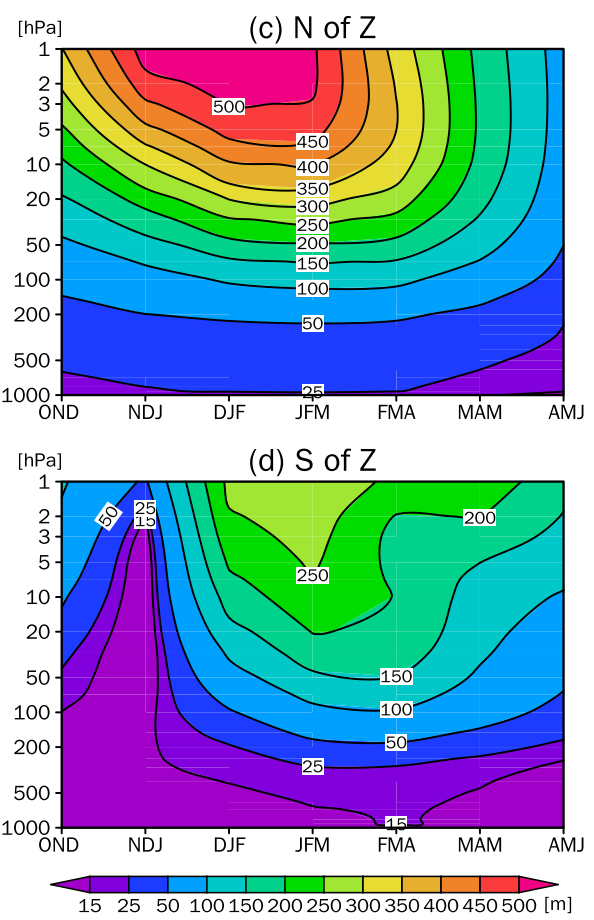

Fig. 2. Time-height cross-sections of $70^{\circ} \mathrm{N}-$ $90^{\circ} \mathrm{N}$ averaged (a) $\sqrt{R}$, (b) ACC, (c) $N$, and (d) $S$ of the zonal mean field of the isobaric surface height $\mathrm{Z}$. The horizontal axis indicates three-month averages (from OND to AMJ). The vertical axis indicates the pressure on a logarithmic scale. The units of $N$ and $S$ are meters. The left (right) color bar is commonly used in the two left (right) panels.
Fig. 3. Time-height cross-sections of $45^{\circ} \mathrm{N}-$ $75^{\circ} \mathrm{N}$ averaged (a) $\sqrt{R}$, (b) $N$, and (c) $S$ of the convergence/divergence of the EP flux. The horizontal axis indicates three-month averages (from OND to AMJ). The vertical axis indicates the pressure on a logarithmic scale. The units of $N$ and $S$ are $\mathrm{m}^{2} \mathrm{~s}^{-2}$. The right color bar is commonly used in the two right panels. 
The seasonality of $\sqrt{R}$ of the EP flux (Fig. 3a) was similar to that of $\sqrt{R}$ of $\mathrm{Z}$ (Fig. 2a). A maximum occurred in the upper stratosphere from JFM to MAM, although a maximum occurred in the lower stratosphere in the case of Z. $N$ of the convergence/ divergence of the EP flux reached a maximum in winter (DJF) and then decreased (Fig. 3b). Local maximum in the upper troposphere (around $200-500 \mathrm{hPa}$ ) is caused by the breaking of the upwardpropagating Rossby waves. The seasonality of the standard deviation of total variability $\left(\sqrt{S^{2}+N^{2}}\right.$; figure omitted) was also similar. Here, in reference to this seasonality, we consider that of $S$ of $Z$. The strength of the polar vortex, which is defined as $Z$ averaged across the polar region in this study, is mainly controlled by the upward propagation of the Rossby waves from the troposphere during the previous several months (e.g., Newman et al. 2001). Thus, the interannual variability of initial anomalies of $\mathrm{Z}$ tends to be large for the JFM and FMA predictions, since the forecasts started in winter when the interannual variability of the upward propagation amount was large and already predicted to some extent. In fact, the maximum of $\mathrm{Z}$ observed during January to February (figure omitted) suggests a large signal in the initial time of prediction (Fig. 2d).

In contrast to the $N$ of the EP flux convergence/divergence, $S$ reached a maximum in early spring (FMA; Fig. 3c), and it is associated with the more deterministically predictable characteristics of Rossby wave propagations in early spring, which may result in a slow decrease in the $S$ of $\mathrm{Z}$ after FMA (Fig. 2d). One potential explanation is that, when the SSW has already occurred at the initial time (January in the case of the forecast of FMA), Rossby waves with large amplitudes forecasted in the troposphere cannot propagate upward into the stratosphere. Since the SSWs occur mainly from December to March (Charlton and Polvani 2007), early spring is the time when the initial stratospheric field can favorably control the Rossby wave propagation, and this further enhances and sustains the signal $(S)$ of the stratospheric circulation Z.

These two relations between the predictability of the convergence/divergence of the EP flux and $\mathrm{Z}$ are illustrated by the seasonality of the predictability at a certain height (Figs. 3 and 2). The seasonalities of the $S$ and $N$ of the convergence/divergence of the EP flux (shown in Figs. 3b and $3 \mathrm{c}$ ) at $10 \mathrm{hPa}$ and $\mathrm{Z}$ (in Figs. $2 \mathrm{c}$ and $2 \mathrm{~d})$ at $50 \mathrm{hPa}(\mathrm{Z} 50)$ are shown in Figs. $4 \mathrm{a}$ and $4 \mathrm{~b}$, respectively. The seasonality of the standard deviation of total variability

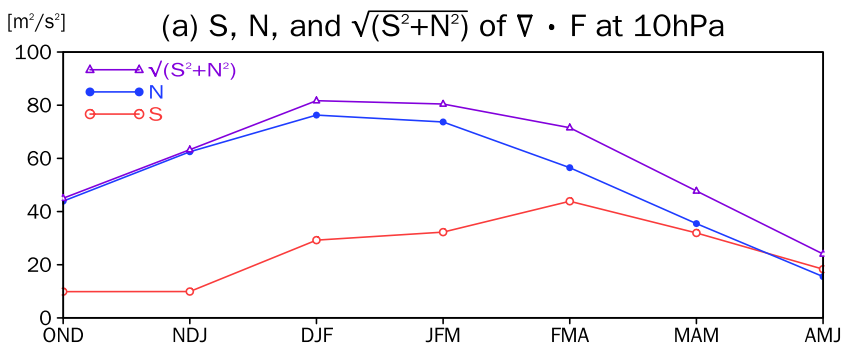

[m]

(b) S and $\mathrm{N}$ of Z50

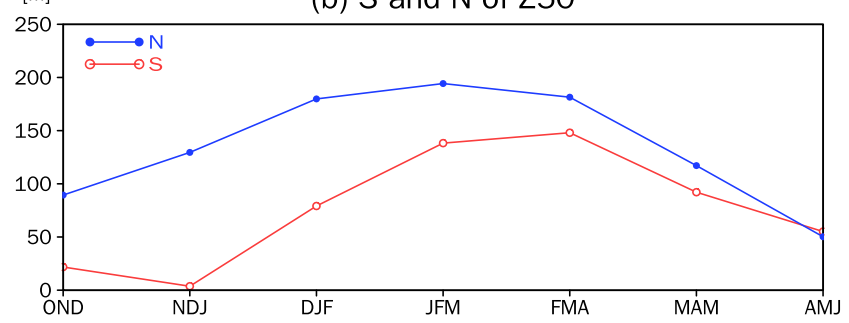

Fig. 4. Seasonalities of (a) $45^{\circ} \mathrm{N}-75^{\circ} \mathrm{N}$ averaged $S$ (red line), $N$ (blue line), and $\sqrt{S^{2}+N^{2}}$ (purple line) of the convergence/divergence of the EP flux at $10 \mathrm{hPa}$ and (b) $70^{\circ} \mathrm{N}-90^{\circ} \mathrm{N}$ averaged $S$ (red line) and $N$ (blue line) of the zonal mean field of $\mathrm{Z}$ at $50 \mathrm{hPa}(\mathrm{Z} 50)$. The horizontal axis indicates the first month of the three-month averages. The unit in the upper panel is $\mathrm{m}^{2} \mathrm{~s}^{-2}$, and that in the lower panel is meters.
$\sqrt{S^{2}+N^{2}}$ is also shown in Fig. 4a. First, the $N$ and $\sqrt{S^{2}+N^{2}}$ of the convergence/divergence of the EP flux reached a maximum in DJF (Fig. 4a), and then, the $N$ of Z50 reached a maximum in JFM (Fig. 4b). The difference of the timings of increase/decrease of $S$ and $N$ presumably corresponds to the fact that the accumulation of upward propagation of Rossby waves affects the stratospheric circulation (Andrews et al. 1987). The $S$ of the convergence/divergence of the EP flux reached a maximum in FMA (Fig. 4a), which is because the changes in the stratospheric circulation, such as the SSW, reversely affect the propagation characteristics of Rossby waves (Andrews et al. 1987).

\section{Quantitative estimate of the downward influence of the signal from the stratosphere to the troposphere}

The results of Subsection 3.3 suggest that high predictability of the stratosphere in late winter is consistent with the seasonality of the upward propagation of Rossby waves. To further understand, we investigated how and to what extent the troposphere is impacted by the high predictability of the stratosphere. Among many theories on downward influence (cf. Kidston et al. 2015), here we refer to AH02's theory, which enables the quantitative estimation of the downward influence of the signal from the stratosphere to the troposphere.

AH02 suggested a two-step mechanism for the downward influence of the stratosphere on the troposphere, in which the stratospheric positive (negative) PV anomaly balances the rising (lowering) of the tropopause height via geostrophic adjustment and then induces the low (high) pressure anomaly at the surface through the stretching (squashing) of the tropospheric vorticity.

With the premise that "the anomaly" in AH02 can be substituted by "the signal" herein and assuming that the signal of the stratosphere is reflected in that of the surface pressure, we quantitatively estimated the predictable signal of the surface pressure anomaly induced by the stratospheric PV signal via the change in the tropopause height. We analyzed the results of the three-month forecasts for FMA. We used the zonal mean PV averaged across $70^{\circ} \mathrm{N}-90^{\circ} \mathrm{N}$ and the same parameter values as those used in AH02.

With the quantitative estimate based on the method in the supplemental material, the surface pressure in balance with the stratospheric PV signal was estimated at $2.51 \mathrm{hPa}$, and it has the same order of magnitude as the actual signal of the surface pressure $(1.13 \mathrm{hPa})$, as was shown previously by Takemura and Maeda (2016). Therefore, the mechanism suggested by AH02 may contribute to the high predictability of sea-level pressure (NAO/ $\mathrm{AO})$. Further analyses are required in order to explain the whole downward influence of the stratosphere on the troposphere.

\section{Conclusion and discussion}

We analyzed the predictability of the NAO using a set of hindcasts from the JMA/MRI-CPS2, focusing on the relation with the stratosphere.

We presented both the predictive skill for the NAO index (ACC) and its indicator $(\sqrt{R})$ increase from late winter to early spring in our seasonal forecasting system. The spatial distribution of the ACC and $\sqrt{R}$ of the zonal mean field of geopotential height $\mathrm{Z}$ in a time-vertical section exhibited areas with high prediction skill and predictability in the stratosphere and near the surface from late winter to early spring. Consistent with this, the high predictability of the convergence/divergence of the Eliassen-Palm flux was also observed in the stratosphere, indicating that the seasonality of upward-propagating Rossby waves contributes to the large signals in the polar vortex variability. Those large signals in the stratosphere are then propagated downward from the stratosphere to the troposphere. On the basis of the mechanism proposed by $\mathrm{AH} 02$, we found that the magnitude of the surface pressure estimated from that of the stratospheric PV anomaly roughly agrees with the actual magnitude of the signal, suggesting that AH02's approach may work. 
In terms of the downward influence of the signal from the stratosphere to the troposphere, we focused only on the mechanism suggested by AH02. However, there might be other mechanisms involved. For example, there is high predictability of the convergence/divergence of the EP flux in the upper troposphere (Fig. 3c), and this might affect the predictability of the meridional circulation in the troposphere and of the circulation near the surface. In other words, the stratospheric circulation affects the propagation characteristics of Rossby waves and then affects the predictability of the circulation near the surface. The convergence/ divergence of the EP flux in the upper troposphere may be influenced by the stratospheric circulation. In this study, we investigated only one of the proposed mechanisms; however, many other theories on the downward influence have been proposed. In order to improve our understanding further, other mechanisms on the downward influence of the signal need to be validated.

This research shows the predictability originating from the stratosphere, especially in early spring. The results imply that, if we improve the reproducibility of the stratospheric circulation by improving forecast systems, the predictive skill of the circulation field near the surface could be further increased.

\section{Acknowledgments}

The authors are grateful to two anonymous reviewers for their constructive comments.

Edited by: R. Kawamura

\section{Supplement}

Supplemental information includes two text sections.

\section{References}

Ambaum, M. H. P., and B. J. Hoskins, 2002: The NAO tropospherestratosphere connection. J. Climate, 15, 1969-1978.

Andrews, D. G., J. R. Holton, and C. B. Leovy, 1987: Middle Atmosphere Dynamics. Academic Press, 489 pp.

Baldwin, M. P., and T. J. Dunkerton, 2001: Stratospheric harbingers of anomalous weather regimes. Science, 294, 581-584.

Barnston, A. G., and R. E. Livezey, 1987: Classification, seasonality and persistence of low-frequency atmospheric circulation patterns. Mon. Wea. Rev., 115, 1083-1126.

Charlton, A. J., and L. M. Polvani, 2007: A new look at stratospheric sudden warmings. Part I: Climatology and modeling benchmarks. J. Climate, 20, 449-469.

Eade, R., D. Smith, A. Scaife, E. Wallace, N. Dunstone, L. Hermanson, and N. Robinson, 2014: Do seasonal-to-decadal climate predictions underestimate the predictability of the real world? Geophys. Res. Lett., 41, 5620-5628.

Edmon, H. J., B. J. Hoskins, and M. E. McIntyre, 1980: EliassenPalm cross sections for the troposphere. J. Atmos. Sci., 37, 2600-2616.

Hitchcock, P., and I. R. Simpson, 2014: The downward influence of stratospheric sudden warmings. J. Atmos. Sci., 71, 38563876.

Hurrell, J. W., 1995: Decadal trends in the North Atlantic Oscillation: Regional temperatures and precipitation. Science, 269, 676-679.

Johansson, A., 2007: Prediction skill of the NAO and PNA from daily to seasonal time scales. J. Climate, 20, 1957-1975.

Kidston, J., A. A. Scaife, S. C. Hardiman, D. M. Mitchell, N. Butchart, M. P. Baldwin, and L. J. Gray, 2015: Stratospheric influence on tropospheric jet streams, storm tracks and surface weather. Nature Geosci., 8, 433-440.
Kobayashi, S., Y. Ota, Y. Harada, A. Ebita, M. Moriya, H. Onoda, K. Onogi, H. Kamahori, C. Kobayashi, H. Endo, and K. Miyaoka, 2015: The JRA-55 reanalysis: General specifications and basic characteristics. J. Meteor. Soc. Japan, 93, $5-48$.

Kodera, K., H. Mukougawa, and S. Itoh, 2008: Tropospheric impact of reflected planetary waves from the stratosphere. Geophys. Res. Lett., 35, L16806.

Kuroda, Y., 2008: Role of the stratosphere on the predictability of medium-range weather forecast: A case study of winter 2003-2004. Geophys. Res. Lett., 35, L19701.

Kuroda, Y., and K. Kodera, 1999: Role of planetary waves in the stratosphere-troposphere coupled variability in the Northern Hemisphere winter. Geophys. Res. Lett., 26, 2375-2378.

Marshall, A. G., and A. A. Scaife, 2010: Improved predictability of stratospheric sudden warming events in an atmospheric general circulation model with enhanced stratospheric resolution. J. Geophys. Res., 115, D16114.

Newman, P. A., E. R. Nash, and J. E. Rosenfield, 2001: What controls the temperature of the Arctic stratosphere during the spring? J. Geophys. Res., 106, 19999-20010.

Palmer, T., and R. Hagedorn, 2006: Predictability of Weather and Climate. Cambridge University Press, 702 pp.

Perlwitz, J., and N. Harnik, 2003: Observational evidence of a stratospheric influence on the troposphere by planetary wave reflection. J. Climate, 16, 3011-3026.

Perlwitz, J., and N. Harnik, 2004: Downward coupling between the stratospheric and troposphere: The relative roles of wave and zonal mean processes. J. Climate, 17, 4902-4909.

Rowell, D. P., C. K. Folland, K. Maskell, and M. N. Ward, 1995: Variability of summer rainfall over tropical north Africa (1906-92): Observations and modelling. Quart. J. Roy. Meteor. Soc., 121, 669-704.

Scaife, A. A., A. Arribas, E. Blockley, A. Brookshaw, R. T. Clark, N. Dunstone, R. Eade, D. Fereday, C. K. Folland, M. Gordon, L. Hermanson, J. R. Knight, D. J. Lea, C. MacLachlan, A. Maidens, M. Martin, A. K. Peterson, D. Smith, M. Vellinga, E. Wallace, J. Waters, and A. Williams, 2014: Skillful long-range prediction of European and North American winters. Geophys. Res. Lett., 41, 2514-2519.

Scaife, A. A., A. Yu. Karpechko, M. P. Baldwin, A. Brookshaw, A. H. Butler, R. Eade, M. Gordon, C. MacLachlan, N. Martin, N. Dunstone, and D. Smith, 2016: Seasonal winter forecasts and the stratosphere. Atmos. Sci. Lett., 17, 51-56.

Sigmond, M., J. F. Scinocca, V. V. Kharin, and T. G. Shepherd, 2013: Enhanced seasonal forecast skill following stratospheric sudden warmings. Nat. Geosci., 6, 98-102.

Sugi, M., R. Kawamura, and N. Sato, 1997: A study of SST-forced variability and potential predictability of seasonal mean fields using the JMA global model. J. Meteor. Soc. Japan, 75, 717-736.

Takaya, Y., S. Hirahara, T. Yasuda, S. Matsueda, T. Toyoda, Y. Fujii, H. Sugimoto, C. Matsukawa, I. Ishikawa, H. Mori, R. Nagasawa, Y. Kubo, N. Adachi, G. Yamanaka, T. Kuragano, A. Shimpo, S. Maeda, and T. Ose, 2017: Japan Meteorological Agency/Meteorological Research Institute-Coupled Prediction System version 2 (JMA/MRI-CPS2): Atmosphereland-ocean-sea ice coupled prediction system. Climate Dyn., doi:10.1007/s00382-017-3638-5.

Takemura, K., and S. Maeda, 2016: Influence of enhanced variability with zonal wavenumber 1 on Arctic Oscillation in late winter to early spring in El Niño conditions. SOLA, 12, $159-164$.

Thompson, D. W. J., and J. M. Wallace, 1998: The Arctic Oscillation signature in the wintertime geopotential height and temperature fields. Geophys. Res. Lett., 25, 1297-1300.

Manuscript received 17 August 2017, accepted 6 October 2017

SOLA: https://www.jstage.jst.go.jp/browse/solal 